\title{
Assistência à Mulher no Pré-Natal, Parto e Nascimento: Contribuições da Rede Cegonha
}

Assistance to Women in Prenatal, Childbirth and Birth: Contributions from the Stork Network

Asistencia a la Mujer en el Prenatal, Parto y Nacimiento: Contribuciones de la Red Cigüeña

Jucelia Salgueiro Nascimento ${ }^{1}$

Maria Rosa da Silva ${ }^{2}$

Elaine Cristina Tôrres Oliveira ${ }^{3}$

Giselle Carlos Santos Brandão Monte ${ }^{4}$

\section{Resumo}

Objetivo: Analisar na literatura, a assistência prestada à mulher no prénatal, parto e nascimento após a implantação da Rede Cegonha. Método: Trata-se de uma revisão integrativa de literatura. A coleta de dados ocorreu em novembro de 2017, sendo realizada na base de dados eletrônica BVS, utilizando os descritores: gestante, Rede Cegonha, trabalho de parto e parto. Foram encontrados 120 artigos, publicados entre 2013 e 2016. Ao final, foram selecionados 9 artigos para a realização desta revisão integrativa. Resultados: A análise dos estudos mostrou que a captação das gestantes ocorre tardiamente; as gestantes não estão

${ }^{1}$ Enfermeira residente na Residência Multiprofissional em Saúde da Família-UNCISAL. Endereço completo: Rua Doutor Jorge de Lima. No 113. Trapiche da Barra. 57010-382. Maceió - AL, Brasil. Email: jueherickson@hotmail.com

${ }^{2}$ Docente do Núcleo Materno-Infantil e do Adolescente da Universidade Estadual de Ciências da Saúde de Alagoas-UNCISAL

${ }^{3}$ Professora auxiliar da Universidade Estadual de Ciências da Saúde de Alagoas-UNCISAL

${ }^{4}$ Mestre em Enfermagem pela UFPE; Docente do Núcleo Materno-Infantil e do Adolescente da Universidade Estadual de Ciências da Saúde - UNCISAL

Recebido: out/2017 - Aceito: Abr/2018. 
tendo acesso aos exames laboratoriais nos serviços de pré-natal de risco habitual e alto risco em tempo; conduta inapropriada de alguns profissionais; desrespeito aos direitos da mulher; vinculação da gestante ao local onde o parto será realizado encontra-se inexistente e presença do enfermeiro obstetra potencializa uma atenção humanizada. Conclusão: Esta revisão permitiu analisar as evidências científicas sobre a assistência prestada à mulher no pré-natal, parto e nascimento após a implantação da Rede Cegonha. Percebeu-se que essa nova estratégia de atenção apresenta alguns avanços significativos, porém precisa superar vários entraves para conseguir atingir seus objetivos.

\section{Descritores: Gestante; Serviços de} Saúde Materno-Infantil; Trabalho de Parto; Parto.

\section{Abstract}

Objective: To analyze in the literature, the assistance given to women in prenatal, childbirth and birth after the implantation of the Stork Network. Method: This is an integrative literature review. The data collect took place in November of 2017, being carried out in the electronic database BVS, using the descriptors: pregnant woman, Stork Network, labor and childbirth. 120 articles were found, published between 2013 and 2016. At the end, 9 articles were selected to carry out this integrative review. Results: The analysis of the studies showed that the uptake of pregnant women occurs late; pregnant women are not having access to laboratory tests in prenatal services of normal risk and high risk in time; inappropriate behavior of some professionals; disregard for women's rights; linking the pregnant woman to the place where the delivery will be performed is non-existent and the presence of the obstetrician nurse enhances humanized attention. Conclusions: This review made it possible to analyze the scientific evidence on the assistance provided to women in prenatal, childbirth and birth after the implantation of the Stork Network. It was noticed that this new strategy of attention presents some significant advances, however it must overcome several obstacles to reach its objectives.

$\begin{array}{lrr}\text { Descriptors: } & \text { Pregnant } & \text { Women; } \\ \text { Maternal-Child } & \text { Health } & \text { Services; }\end{array}$ Labor, Obstetric; Parturition.

\section{Resumen}


Objetivo: Analizar en la literatura, la asistencia prestada a la mujer en el prenatal, parto y nacimiento después de la implantación de la Red Cigüeña. Método: Se trata de una revisión integrativa de literatura. La recolección de datos ocurrió en noviembre de 2017, siendo realizada en la base de datos electrónica BVS, utilizando los descriptores: gestante, Red Cigüeña, trabajo de parto y parto. Se encontraron 120 artículos, publicados entre 2013 y 2016. Al final, se seleccionaron 9 artículos para la realización de esta revisión integrativa. Resultados: El análisis de los estudios mostró que la captación de las gestantes ocurre tardíamente; las gestantes no están teniendo acceso a los exámenes de laboratorio en los servicios de prenatal de riesgo habitual $y$ alto riesgo en tiempo; conducta inapropiada de algunos profesionales; incumplimiento de los derechos de la mujer; la vinculación de la gestante al lugar donde el parto será realizado se encuentra inexistente y la presencia del enfermero obstetra potencializa una atención humanizada. Conclusiones: Esta revisión permitió analizar las evidencias cientificas sobre la asistencia prestada a la mujer en el prenatal, parto y nacimiento después de la implantación de la Red Cigüeña. Se percibió que esta nueva estrategia de atención presenta algunos avances significativos, pero necesita superar varios obstáculos para alcanzar sus objetivos.

Descriptores: Mujeres Embarazadas; Servicios de Salud Materno-Infantil; Trabajo de Parto; Parto.

\section{Introdução}

No início do século $\mathrm{XX}$ os programas voltados para as mulheres eram limitados ao período gestacional, garantiam apenas, a gravidez e o parto seguro. Em 1983 as ações programáticas apresentadas na Comissão Parlamentar Mista de Inquérito (CPMI) pelo Ministério da Saúde (MS) baseavam-se no controle da natalidade. Por meio de movimentos e organizações feministas ocorre uma ruptura de paradigma materno infantil onde a mulher era vista como reprodutora e produtora de força de trabalho incorporando o conceito de integralidade à saúde da mulher ${ }^{(1)}$.

No ano de 1983 o MS, atendendo às reivindicações dos movimentos de mulheres, elaborou o Programa de Assistência Integral à Saúde da Mulher (PAISM), que só foi amplamente 
divulgado em 1984. Assim, o PAISM começa a modificar o foco das ações, além de eleger as prioridades diferenciadas naquela linha de cuidado. Desta forma, houve uma quebra paradigmática relativa aos ideais norteadores das políticas de saúde das mulheres $^{(2)}$.

Baseado no conceito de atenção integral à saúde das mulheres, o PAISM rompeu com a visão tradicional acerca das necessidades de saúde da mulher, restrita ao seu papel reprodutivo dentro do contexto familiar, sobretudo no âmbito da medicina, descentralizando o atendimento às mulheres a questões relativas apenas à reprodução ${ }^{(2,3)}$. Dessa forma, o PAISM sofreu grande influência da implantação e implementação do Sistema Único de Saúde (SUS), por meio da incorporação de seus princípios e diretrizes, até na sua organização sistemática ${ }^{(4)}$.

O PAISM incorporou como princípios e diretrizes as propostas de descentralização, hierarquização e regionalização dos serviços, bem como a integralidade e a equidade da atenção à saúde da mulher. Abrangia ações educativas, preventivas, de diagnóstico, tratamento e recuperação, englobando a assistência à mulher em clínica ginecológica, no pré-natal, parto e puerpério. Contemplava também ações relativas ao climatério, planejamento familiar, tratamento de doenças sexualmente transmissíveis - DST, câncer de colo de útero e de mama, além do atendimento de outras inúmeras necessidades identificadas a partir do perfil populacional das mulheres brasileiras $^{(2)}$.

Em 2011, o MS implanta um novo modelo de atenção de âmbito nacional operacionalizado pelo SUS e instituído pelo artigo $1^{\mathrm{o}}$ da Portaria $\mathrm{n}^{\mathrm{o}}$ 1.459/GM/MS. Intitulado Rede Cegonha, trata-se de uma rede de cuidados que objetiva garantir à mulher os direitos ao planejamento reprodutivo, e à atenção humanizada durante a gravidez, ao parto e puerpério, mas também assegurar o direito ao nascimento seguro e desenvolvimento saudável da criança ${ }^{(5)}$.

Esse modelo de atenção está fundamentado nos princípios da humanização da assistência. E segue os seus antecedentes buscando garantir os direitos assegurados à mulher, ao recém-nascido e à criança; ampliar o acesso; acolhimento e qualidade da atenção pré-natal; subsidiar o transporte de urgência e emergência; promover a realização de vinculação da gestante a uma instituição de referência para assistência ao parto; incentivar a realização de parto e nascimento 
seguros, por meio da utilização de boas práticas obstétricas de atenção recomendadas pela Organização Mundial da Saúde (OMS); apoiar a presença de acompanhante de escolha da mulher durante o parto; garantir o acesso ao planejamento reprodutivo; e assistência de qualidade e resolutiva à criança de zero a 24 meses de vida ${ }^{(5)}$.

A conjectura da Rede Cegonha esclarece que quando totalmente implantado este atual modelo de atenção visa oferecer, ainda, subsídio para o funcionamento dos sistemas locais. Isto mediante $\mathrm{o}$ incentivo $\mathrm{e}$ financiamento de transporte sanitário, regulação, e estrutura física. Estão previstas mudanças relativas à melhoria da estrutura dos serviços, criação de casas de gestante e centros de parto normal, aumento do número de leitos de Unidade de Terapia Intensiva (UTI) neonatal e adulta, melhoria nos serviços ambulatoriais e de transporte, todas com incentivos financeiros. $\mathrm{O}$ conjunto dessas mudanças contribuirá para que Rede Cegonha realmente seja efetivada $^{(5)}$.

A atenção ao período gravídicopuerperal é um ponto crucial no cuidado integral à saúde da mulher. Sem minimizar os fatores que estão envoltos ao mundo feminino, a importância aos cuidados gravídicos puerperais se destaca, principalmente, relacionada à redução dos indicadores de morbimortalidade materna e neonatal, os quais refletem a qualidade dos serviços prestados à mulher e ao recém$\operatorname{nascido}^{(6)}$.

Uma vez que a qualidade da assistência mantém relação direta com os níveis de saúde de mães e conceptos, a atenção pré-natal e puerperal implica ser organizada de forma a contemplar a integralidade e humanização no cuidado. Logo, deve atender às reais necessidades das mulheres durante os períodos gestacional e puerperal, estar embasada em conhecimentos técnicocientíficos atualizados e servir-se de meios e recursos adequados ao cuidado específico de cada mulher ${ }^{(7)}$.

Nas últimas décadas, com o auxílio do governo e da sociedade, o Brasil progrediu muito no atendimento à mulher e à criança. Dados do MS apontam que, entre o período de 1990 e 2010, o Brasil reduziu a Razão de Mortalidade Materna (RMM) de 141 para 68 óbitos maternos por 100 mil nascidos vivos, o que representa uma redução de $51 \%{ }^{(8)}$. Em relação à mortalidade infantil, houve decréscimo de cerca de $40 \%$ desses óbitos nas últimas duas décadas. Em 1990, a mortalidade infantil era de 26,6 óbitos por 1000 nascidos vivos, e em 2010, de 
16,2 óbitos por 1000 nascidos vivos. Apesar do esforço, os níveis da mortalidade permanecem elevados quando comparados com os níveis de outros países com semelhantes índices de desenvolvimento econômico ${ }^{(9)}$.

Desta forma, a atenção pré-natal e a perinatal, de qualidade, contribuem para a redução da mortalidade materna, bem como para a redução da mortalidade infantil. Entretanto apesar dos avanços na melhoria da qualidade mobilizados pelas políticas públicas de atenção à saúde da mulher, a redução da mortalidade materna e infantil permanece desafiadora $^{(2)}$.

Diante desse contexto, considerando a relevância da Rede Cegonha e seu papel na estruturação e organização da atenção à saúde materno-infantil no Brasil e principalmente, na redução da morbimortalidade materna e infantil, surge $o$ interesse de conhecer a realidade da assistência prestada as gestantes. Assim, esse estudo tem como objetivo identificar as evidências científicas sobre a assistência prestada à mulher no pré-natal, parto e nascimento após a implantação da Rede Cegonha, no período de 2012 a 2016.

\section{Método}

$\mathrm{O}$ presente estudo consiste em uma revisão integrativa de literatura, a qual se constitui de um estudo com coleta de dados realizada a partir de fontes secundárias. Este método tem a finalidade de reunir e sistematizar resultados de pesquisas sobre um delimitado tema ou questão de maneira sistemática e ordenada, contribuindo para 0 aprofundamento do conhecimento do tema investigado ${ }^{(10)}$. Tendo como questão norteadora: quais são as evidências científicas sobre a assistência prestada à mulher no prénatal, parto e nascimento após a implantação da Rede Cegonha?

As etapas seguidas na pesquisa foram as seguintes: delimitação do tema e definição da questão da pesquisa; busca nas bases de dados utilizando os seguintes descritores: gestante, rede cegonha, trabalho de parto e parto. Após coleta dos dados passou-se para a categorização dos estudos e análise dos dados. E por fim, a discussão e apresentação dos resultados obtidos.

A coleta de dados ocorreu no mês de novembro de 2017. Inicialmente foi realizada a partir de buscas nas bases de dados do Scielo (Scientific Eletronic Library Online), Lilacs (Literatura Latino-Americana e do Caribe em Ciências da Saúde), Medline (Medical Literature Analysis and Retrieval 
System Online), BDE- Enfermagem

(Base de Dados de Enfermagem). Utilizando como critérios de inclusão: artigos completos nos idiomas português e inglês, publicados no período de 2012 a 2016, gratuitos, que contivessem em seus títulos e/ou resumos algumas das palavras chaves definidas nesta pesquisa. E os critérios de exclusão foram: estudos duplicados nas diferentes bases de dados ou que não atendesse aos objetivos explicita da pesquisa.

Para a realização da busca pelos estudos, foram utilizadas as seguintes combinações entre os descritores da pesquisa: "rede cegonha" AND gestante; "rede cegonha" AND parto AND "trabalho de parto"; gestante AND "rede cegonha" AND "pré-natal". Nesta busca foram identificados no site Biblioteca Virtual em Saúde (BVS) 120 estudos. Após realizar leitura detalhada dos títulos e resumos dos artigos, 29 foram excluídos por não tratarem da temática abordada na pesquisa; 13 foram excluídos por estarem duplicados em diferentes bases de dados; 13 excluídos por não conterem em seus títulos e resumos, os descritores do estudo; 9 excluídos por não estarem disponíveis na íntegra; 8 excluídos por se tratarem de documentos que não correspondiam a metodologia abordada e 20 excluídos por estarem disponíveis em outros idiomas. Ao final, foram selecionados 9 artigos para a realização desta revisão integrativa de literatura, por apresentarem aspectos que respondiam à questão norteadora.

Para a seleção das publicações, primeiramente foram lidos todos os títulos dos estudos. Nas publicações em que os títulos possuíam algum descritor e/ou palavras que remetessem ao objetivo da revisão integrativa, os resumos foram analisados. Os resultados obtidos foram analisados através do formulário para coleta de dados, os quais foram dispostos em tabelas e quadros.

A organização e tabulação dos dados se deram por meio de um instrumento de coleta de dados contendo: autor, ano de publicação, título do artigo, objetivos, principais resultados e conclusão. Realizou-se a análise dos artigos, em seguida foram extraídos os conceitos abordados em cada artigo que atendessem ao objetivo do estudo.

E por fim, a última etapa, composta pela discussão e análise dos resultados, sendo apresentada de forma descritiva, possibilitando a identificação das evidências e a necessidade de outras pesquisas. 


\section{Resultados}

Quanto à caracterização dos nove artigos selecionados, em relação ao ano de publicação, dois $(22,22 \%)$ foram publicados em 2013, dois $(22,22 \%)$ em 2014 e cinco em 2016. Quanto à área de conhecimento, houve prevalência do campo da Enfermagem, com cinco artigos $(55,55 \%)$, seguidos por Saúde Pública, com dois artigos $(22,22 \%)$ e Saúde Coletiva e Médica, com um artigo $(11,11 \%)$ cada.

Os nove artigos se encontram publicados em oito revistas. Dentre as oito, três $(37,5 \%)$ são da Enfermagem, duas (25\%) são de Saúde Coletiva, uma (12,5\%) de Saúde Pública, uma (12,5\%) de Comunicação e Educação e uma $(12,5 \%)$ de Medicina. Quanto ao idioma dos artigos, seis $(66,66 \%)$ estão em português e três $(33,33 \%)$ em inglês.

Após a leitura exaustiva dos artigos selecionados, houve a construção de um quadro contendo a identificação do artigo representado pela letra A (artigo) seguido pelo número cardinal, autores, título, ano de publicação e periódico publicado. Segue a baixo o quadro 1 , com a sistematização dos artigos selecionados.

\section{Quadro 1- Sistematização dos Artigos Selecionados para a Pesquisa Atual}

\begin{tabular}{|c|c|c|c|c|}
\hline Identificação & $\begin{array}{l}\text { Autores e Ano de } \\
\text { publicação }\end{array}$ & Título & $\begin{array}{c}\text { Ano de } \\
\text { publicação }\end{array}$ & Periódico \\
\hline A1 & $\begin{array}{l}\text { Cavalcante } \text { PCS, Junior } \\
\text { GDG, Vasconcellos } \\
\text { ALR, Guerrero. }\end{array}$ & Um modelo lógico da Rede Cegonha. & 2013 & $\begin{array}{l}\text { Physis } \\
\text { Revista de } \\
\text { Saúde } \\
\text { Coletiva. }\end{array}$ \\
\hline A2 & $\begin{array}{l}\text { Martinelli KG, Neto } \\
\text { ETS, Gama SGN, } \\
\text { Oliveira AE. }\end{array}$ & $\begin{array}{l}\text { Adequação do processo da assistência } \\
\text { pré-natal segundo os critérios do } \\
\text { Programa de Humanização do Pré- } \\
\text { natal e Nascimento e Rede Cegonha. }\end{array}$ & 2014 & $\begin{array}{l}\text { Rev. Bras. } \\
\text { Ginecologia } \\
\text { e Obstetrícia. }\end{array}$ \\
\hline A3 & $\begin{array}{l}\text { Carvalho VF, Kerber } \\
\text { NPC, Azambuja EP, } \\
\text { Bueno FF, Silveira RS, } \\
\text { Barros AM. }\end{array}$ & 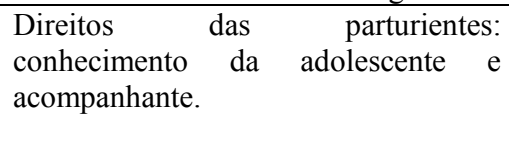 & 2014 & $\begin{array}{l}\text { Saúde e } \\
\text { Sociedade. }\end{array}$ \\
\hline A4 & $\begin{array}{l}\text { Souza AMM, Souza KV, } \\
\text { Rezende EM, Martins } \\
\text { EF, Campos D, Lansky } \\
\text { S. }\end{array}$ & $\begin{array}{l}\text { Práticas na assistência ao parto em } \\
\text { maternidades com inserção de } \\
\text { enfermeiras obstétricas, em Belo } \\
\text { Horizonte, Minas Gerais. }\end{array}$ & 2016 & $\begin{array}{l}\text { Esc. Anna } \\
\text { Nery. }\end{array}$ \\
\hline A5 & $\begin{array}{llr}\text { Andrade } & \text { SMAS, } \\
\text { Manzotti } & \text { CAS, Gouvêa } \\
\text { JAG, Gomes CF, } \\
\text { Bernuci } & \text { MP, Cassiano } \\
\text { ACM. } & \end{array}$ & $\begin{array}{lccr}\text { Impacto da implantação da } & \text { Rede } \\
\text { Cegonha nas } & \text { hospitalizações em } \\
\text { Unidade de } & \text { Terapia Intensiva } \\
\text { Neonatal. } & & & \end{array}$ & 2016 & Rev. Rene. \\
\hline A6 & $\begin{array}{l}\text { Oliveira G, Bertole CFC, } \\
\text { Prates LA, Astarita KB, } \\
\text { Silva TC, Rossel LB. }\end{array}$ & $\begin{array}{l}\text { Acesso ao pré-natal no âmbito da } \\
\text { atenção básica: revisão integrativa. }\end{array}$ & 2016 & $\begin{array}{l}\text { J Nurs UFPE } \\
\text { on line. }\end{array}$ \\
\hline A7 & $\begin{array}{l}\text { Oliveira JDG, Campo } \\
\text { TNC, Souza FMLC, } \\
\text { Davim RMB, Dantas JC. }\end{array}$ & $\begin{array}{l}\text { Percepção de enfermeiros obstetras na } \\
\text { assistência à parturiente. }\end{array}$ & 2016 & $\begin{array}{l}\text { Journal of } \\
\text { Nursing. }\end{array}$ \\
\hline A8 & $\begin{array}{l}\text { Xavier FA, Moreira } \\
\text { LMS, Costa ICC. }\end{array}$ & $\begin{array}{l}\text { O direito de nascer com dignidade no } \\
\text { Rio Grande do Norte: um } \\
\text { compromisso do Ministério Público }\end{array}$ & 2016 & $\begin{array}{l}\text { Revista } \\
\text { Ciência } \\
\text { Plural. }\end{array}$ \\
\hline
\end{tabular}




\begin{tabular}{|l|l|l|l|l|}
\hline & & Estadual. & & \\
\hline A9 & Carneiro RG. & $\begin{array}{l}\text { Dilemas antropológicos de uma } \\
\text { agenda de saúde pública: programa } \\
\text { Rede Cegonha, pessoalidade e } \\
\text { pluralidade. }\end{array}$ & 2013 & Interface. \\
\hline
\end{tabular}

Percebeu-se que quanto aos temas abordados nos artigos, três $(33,33 \%)$ trazem a temática sobre o momento do trabalho de parto e parto, três $(33,33 \%)$ falam sobre a assistência prestada no pré-natal, um $(11,11 \%)$ trata do processo de gestão da Rede Cegonha, um $(11,11 \%)$ trás uma reflexão sobre o papel da mulher nessa estratégia de atenção à saúde e um (11,11\%) sobre a assistência prestada ao recém-nascido. Abaixo, segue a tabela 1, que descreve a caracterização dos artigos incluídos na revisão integrativa de acordo com os eixos temáticos, e seus respectivos números de associação. Maceió-AL, 2017.

Tabela 1. Eixos temáticos/Número de Publicações/Referências

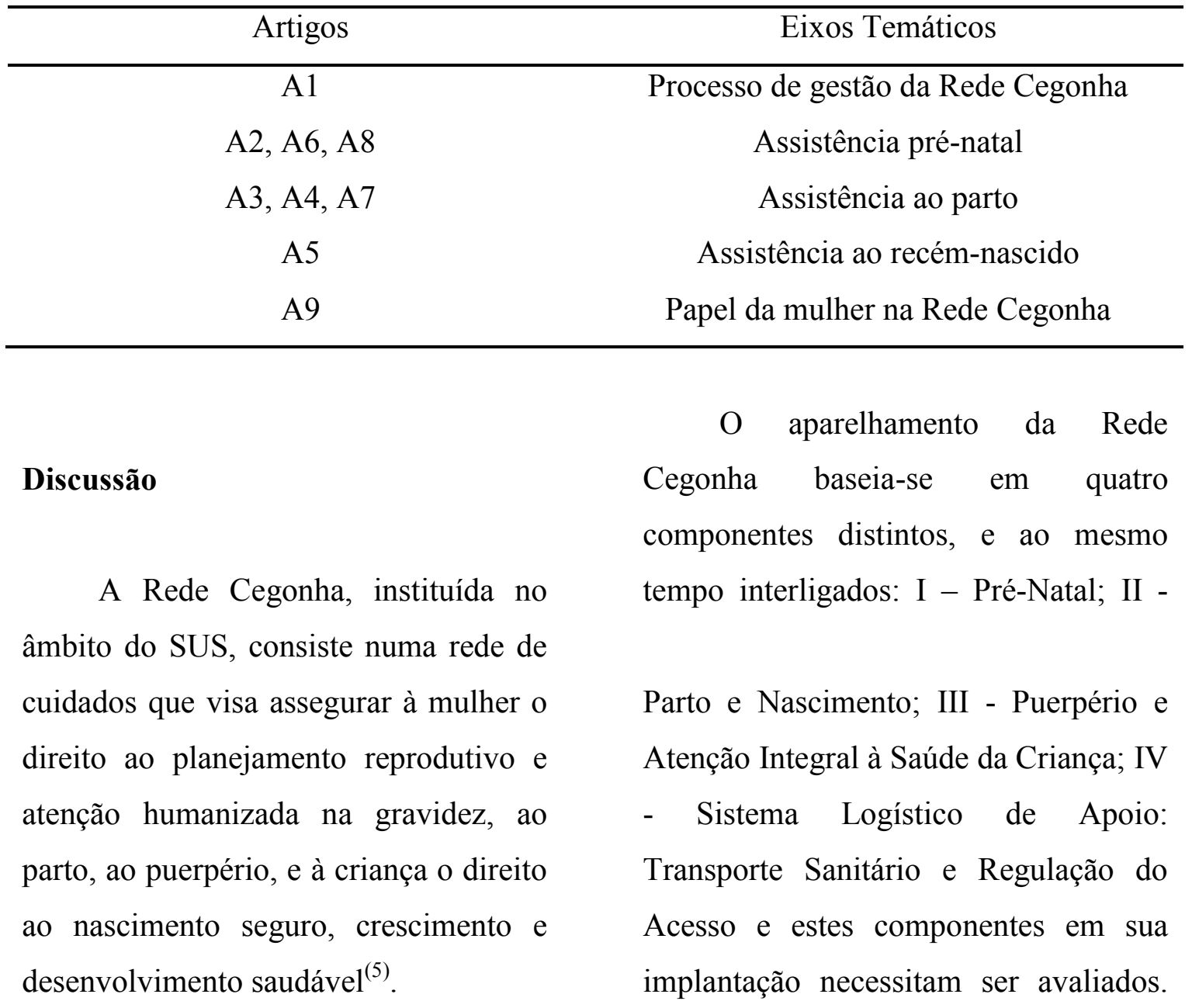


Cada componente compreende uma gama de ações de atenção à saúde ${ }^{(5)}$.

Então, ao analisar os artigos selecionados nesse estudo, a literatura aborda, principalmente, dois componentes da Rede Cegonha: I- Prénatal e II- Parto e Nascimento.

\section{Componente I- Pré-Natal}

As mortalidades materna e infantil têm sido objeto de interesse ao longo da história da saúde coletiva no nosso país e várias estratégias têm sido propostas para a resolução dessa problemática ${ }^{(11)}$. Melhorar a saúde materna e impedir mortes evitáveis, ainda é um dos maiores objetivos nacionais e internacionais no campo da saúde e dos direitos reprodutivos a serem alcançados, no qual se discutem quais medidas necessárias e eficazes para alcançar tais propósitos ${ }^{(12)}$.

A Rede Cegonha preconiza que o início do pré-natal seja precoce, que a assistência seja prestada com qualidade, acesso ao pré-natal de alto risco em tempo oportuno, realização dos exames do pré-natal de risco habitual e alto risco e acesso aos resultados em tempo oportuno, e vinculação da gestante desde o pré-natal ao local onde acontecerá o parto ${ }^{(5)}$. Porém, os estudos analisados nessa pesquisa apresentam divergência quanto ao que é recomendado por essa estratégia de atenção à saúde da mulher.

Oliveira et al. ${ }^{(13)}$ constataram em seu estudo que houve captação tardia das mulheres grávidas e atraso na programação das consultas, o que acaba comprometendo a qualidade da assistência prestada. Ao iniciar o prénatal tardiamente, perde-se a oportunidade de fazer um bom acompanhamento da gestação e isso acaba contribuindo para o surgimento de possíveis complicações que poderiam ser evitadas ou minimizadas.

Os estudos de Martinelle et al. ${ }^{(14)}$ e Xavier, Moreira, Costa $^{(15)}$ corroboram entre si, ao identificarem que as gestantes não estão tendo acesso aos exames laboratoriais nos serviços de pré-natal de risco habitual e alto risco em tempo oportuno. A falta de acesso a esses exames podem contribuir para o aumento das complicações durante o parto e o puerpério, tais como infecções urinárias, hemorragias e más formações que podem resultar em óbitos maternos e infantis ${ }^{(16)}$. Infelizmente, essa situação acaba comprometendo a qualidade da assistência prestada.

Além da dificuldade de acesso aos exames laboratoriais, Martinelli et al. ${ }^{(14)}$ verificaram que a realização dos teste rápidos apresentam índices bem a baixo 
do que é recomendado pela RC. Outros aspectos importantes da assistência prénatal também foram evidenciados nesse estudo, a baixa cobertura de vacinação antitetânica e a ausência de ações educativas sobre o aleitamento materno. A RC trouxe como algo inovador a realização dos testes rápidos, com $\mathrm{o}$ intuito de otimizar a assistência. Porém, na prática, não está conseguindo atingir seu objetivo.

Xavier, Moreira, Costa ${ }^{(15)}$ constataram também em seu estudo que a vinculação da gestante ao local onde o parto será realizado encontra-se inexistente. As gestantes continuam peregrinando entre maternidades superlotadas e sem condições de atendêlas. A prática da peregrinação se mostra como fonte potencial de mortes de mães e bebês e necessita de urgente atenção das autoridades de saúde.

A falta de leitos obstétricos é uma realidade generalizada no país. Eles apresentam-se como poucas opções e podem se configurar em pontos de estrangulamento ou oportunidades de desconcentração e regionalizações alternativas $^{(17)}$. Fatores como dificuldade no acesso à maternidade, desorganização do sistema de saúde na oferta de leitos obstétricos e neonatais, além da precariedade na infraestrutura hospitalar, contribuem para a baixa qualidade na assistência obstétrica e perinatal no Brasil ${ }^{(18)}$.

A avaliação dos parâmetros que compõem a Rede Cegonha permite inferir diretamente a qualidade da assistência pré-natal nos serviços do SUS. Sendo assim, as baixas taxas de adequação do processo pré-natal nos vários níveis pode ter resultado em desfechos indesejáveis como nascimento de crianças prematuras e de baixo peso, além de contribuir para mortalidade materna e perinatal ${ }^{(14)}$.

\section{Componente II- Parto e Nascimento}

No Brasil, testemunha-se, o que se pode denominar de um movimento de transição do modelo de assistência ao parto e nascimento. $\mathrm{O}$ foco da mudança parte de um modelo que considera o parto um evento médico e de risco, cujo cenário é o ambiente hospitalar. Em detrimento do cuidado centrado na mulher, no seu protagonismo e autonomia $^{(19)}$. Segundo Davis-Floyd ${ }^{(20)}$, esse modelo de atenção à saúde, denominado tecnocrático, é marcado por intervenções desnecessárias e prejudicais que resultam em altas taxas de cesarianas, e considera o nascimento um evento patológico que precisa ser tratado. 
Nesse contexto, a partir da década de 1980, ganhou visibilidade o movimento da humanização do parto que, além da assistência acolhedora e respeitosa à parturiente, propôs a assistência baseada em evidências científicas, um dos marcos mais importantes da transição para mudança do modelo assistencial obstétrico brasileiro $^{(19)}$.

Assim, a Rede Cegonha representa um conjunto de iniciativas que envolvem mudanças: (a) no processo de cuidado à gravidez, ao parto e ao nascimento; (b) na articulação dos pontos de atenção em rede e regulação obstétrica no momento do parto; (c) na qualificação técnica das equipes de atenção primária e no âmbito das maternidades; (d) na melhoria da ambiência dos serviços de saúde (UBS e maternidades); (e) na ampliação de serviços e profissionais, para estimular a prática do parto fisiológico; e (f) na humanização do parto e do nascimento (Casa de Parto Normal, enfermeira obstétrica, parteiras, Casa da Mãe e do Bebê) ${ }^{(21)}$.

$\mathrm{Na}$ análise dos estudos selecionados para este componente, pode-se observar que a assistência prestada à parturiente vem caminhando para um modelo de atenção pautado nos princípios da humanização e adesão as boas práticas de atenção ao parto $\mathrm{e}$ nascimento. Este cenário pôde ser visualizado nos trabalhos de Souza et al. ${ }^{(19)}$ e Oliveira et al. ${ }^{(22)}$.

A prática obstétrica baseada em evidências descrita pela $\mathrm{OMS}^{(23)} \mathrm{e}$, mais tarde, corroborada pelo $\mathrm{MS}^{(5)}$ tem como base a classificação de condutas obstétricas no parto normal, a partir de evidências científicas, segundo critérios de utilidade, eficácia e risco. Esses documentos destacam, ainda, a enfermeira obstétrica como componente fundamental na assistência humanizada ao parto.

Souza et al. ${ }^{(19)}$ e Oliveira et al. ${ }^{(22)}$ afirmam que o predomínio dessas profissionais na assistência obstétrica reforça a sua importante contribuição no que se refere à prática assistencial, em concordância com a OMS, o MS e os princípios da humanização. Ao mesmo tempo, propõe que elas têm peso importante quando determinadas práticas obstétricas são ou não respeitadas.

Contudo, Carvalho et al. ${ }^{(24)} \mathrm{e}$ Carneiro $^{(25)}$ trazem em seus estudos uma realidade diferente, na qual há predomínio de práticas assistências marcadas pelo desrespeito aos direitos da parturiente, condutas inapropriadas, falta de infraestrutura adequada dos serviços obstétricos, número de leitos 
insuficientes para supri a demanda e despreparo de alguns profissionais. Tal situação está contribuindo para um desfeche desastroso tanto para a mulher quanto para o recém-nascido.

Para a concretização de atenção de qualidade e humanizada, é dever dos serviços e profissionais de saúde acolher com dignidade a mulher e o recém-nascido, enfocando-os como sujeitos de direitos. Esse tipo de atenção depende da provisão dos recursos, da organização de rotinas com procedimentos comprovadamente benéficos, evitando-se intervenções desnecessárias, e do estabelecimento de relações baseadas em princípios éticos, garantindo-se a privacidade, a autonomia e o compartilhamento com a mulher e sua família das decisões sobre as condutas a serem adotadas ${ }^{(26)}$.

Ainda nessa perspectiva, Cavalcanti et al. ${ }^{(27)}$ enfatizam a necessidade de se trabalhar com a perspectiva de que os "pontos de atenção" necessários ao cuidado materno-infantil devem estar conectados e funcionar em rede, minimizando, assim, a fragmentação, o desperdício de recurso e a ineficiência, ao mesmo tempo em que busca garantir a integralidade da assistência.

\section{Conclusão}

As evidências científicas pesquisadas na literatura mostram que após a implantação da Rede Cegonha, houve avanços significativos, porém ainda há entraves. Como avanços podese observar o incentivo ao parto normal e investimento na atuação do enfermeiro obstetra, promovendo uma assistência humaniza a mulher. E dentre os entraves pode ser encontrado atrasos na captação das gestantes e atraso na realização dos exames, inexistência da vinculação da gestante ao local onde acontecerá o parto, conduta inapropriada de alguns profissionais, realização de testes rápidos aquém do que é preconizado.

É importante frisar, que a Rede Cegonha é uma estratégia relativamente recente e, por essa razão, não existem muitos trabalhos abordando essa temática. Assim, faz-se necessário que outros estudos possam ser realizados para que se possa avaliar a qualidade da assistência prestada à mulher.

Além disso, a Rede Cegonha foi criada pelo Governo Federal, porém os municípios precisam aderir a esta nova forma de planejamento das ações em saúde materno-infantil. Desta forma, se os gestores municipais não estiverem sensíveis à causa, a qualidade da assistência realizada continuará sendo 
realizada de forma fragmentada $\mathrm{e}$ desorganizada, contribuindo para que as taxas de mortalidade materna e infantil continuem em níveis elevados no Brasil.

Portanto, para que a Rede Cegonha consiga atingir seus objetivos, faz-se necessário uma ação conjunta entre a sociedade civil, profissionais da saúde e o poder público, agindo com o propósito de melhorar a qualidade da assistência prestada à mulher no período gravídico puerperal.

\section{Referências}

1. Ministério da Saúde (BR). Secretaria de atenção à Saúde. Departamento de Ações Programáticas Estratégicas. Direitos sexuais e direitos reprodutivos: uma prioridade do governo. Brasília: Ministério da Saúde; 2005.

2. Ministério da Saúde (BR). Política nacional de atenção integral à saúde da mulher: princípios e diretrizes. Brasília: Ministério da Saúde; 2009.

3. Ministério da Saúde (BR). Secretaria de Atenção à Saúde. Departamento de Ações Programáticas Estratégicas. Política nacional de atenção integral à saúde da mulher: plano de ação 2004-2007 / Ministério da Saúde, Secretaria de Atenção à Saúde, Departamento de Ações Programáticas Estratégicas. Brasília: Ministério da Saúde; 2004.

4. Ministério da Saúde (BR). Portaria n 373, de 27 de fevereiro de 2002. Norma Operacional da Assistência à Saúde- NOAS-SUS 01/02. Diário Oficial [da] República. Brasília: Ministério da Saúde; 2002.
5. Ministério da Saúde (BR). Portaria $n^{\circ} 1.459$, de 24 de junho de 2011 Institui, no âmbito do Sistema Único de Saúde - SUS - a Rede Cegonha. Diário Ofícial da República. Brasília: Ministério da Saúde; 2011.

6. Oliveira, FAM. Avaliação da atenção perinatal em maternidades de risco habitual em município do sul do Brasil [tese mestrado]. Curitiba: Programa de Pós-graduação em Enfermagem da Universidade Federal do Paraná; 2015. Disponível em: http://dspace.c3sl.ufpr.br:8080/dspace/ bitstream/handle/1884/37962/R\%20$\% 20 \mathrm{D} \% 20 \% 20 \mathrm{FABIO} \% 20 \mathrm{ANDRE} \%$ 20MIRANDA\%20DE\%20OLIVEIRA. pdf? sequence $=3 \&$ isAllowed $=y$.

7. Ministério da Saúde (BR). Pré-natal e Puerpério: Atenção qualificada e humanizada. 5. ed. Brasília: Ministério da Saúde; 2006.

8. Ministério da Saúde (BR). Secretaria de Vigilância em Saúde. Saúde Brasil 2011: uma análise da situação de saúde e a vigilância da saúde da mulher. Brasília: Ministério da Saúde; 2011c.

9. Ministério da Saúde (BR). Secretaria de Vigilância em Saúde. Saúde Brasil 2011: uma análise da situação e de evidências selecionadas de impacto de ações de vigilância em saúde. Brasília: Ministério da Saúde, 2011d.

10. Mendes KDS, Silveira RCCP, Galvão CM. Revisão integrativa: método de pesquisa para incorporação de evidências na saúde e na Enfermagem. Enfermagem (Florianópolis) [periódico na Internet]. 2008 [citado 2017 jun. 01]; 17(4):75864. Disponível em: http://www.scielo. br/pdf/tce/v17n4/18.pdf. 
11. Fernandes, RZS. Integração de práticas de vigilância sanitária e práticas assistenciais na rede cegonha: a busca pela integralidade do cuidado [tese de mestrado]. Campinas: Programa de Pós-graduação em Saúde Coletiva, Política e Gestão em Saúde da Faculdade de Ciências Médicas da Universidade de Estadual de Campinas; 2014. Disponível em: http://www.bibliotecadigital.unicamp.b $\mathrm{r} /$ document $/$ ? code $=000942738 \& \mathrm{fd}=\mathrm{y}$.

12. Pitanguy J. Parte I - História, política e conceitos: do movimento nacional e internacional de saúde e direitos reprodutivos. Rio de Janeiro; Editora FIOCRUZ; 2013: 19-3.

13. Oliveira G, Bortole CFC, Prates LA, Astarita KB, Silva TC, Rossel LB. Access to prenatal care within the primary care: an integrative review. Enfermagem (Recife) [periódico na Internet]. 2016 [citado 2017 jun. 09]; 10(9):3446-54. Disponível em: https://periodicos.ufpe.br/revistas/revis taenfermagem/article/view/11427

14. Martinelli KG, Neto ETS, Gama SGN, Oliveira AE. Adequação do processo da assistência pré-natal segundo os critérios do Programa de Humanização do Pré-natal e Nascimento e Rede Cegonha. Rev. Bras. Ginecologia e Obstetrícia (Rio de Janeiro) [periódico na Internet]. 2014 [citado 2017 jun. 18]; 36(2):56-4. Disponível em: http://www.scielo.br/ pdf/rbgo/v36n2/0100-7203-rbgo-36-02 -00056.pdf.

15. Xavier FA, Moreira LMS, Costa ICC. O direito de nascer com dignidade no Rio Grande do Norte: um compromisso do Ministério Público Estadual. Saúde Coletiva (Natal) [periódico na Internet]. 2016 [citado 2017 jun. 27]; 2(2): 55-66. Disponível em: https://periodicos.ufrn.br/rcp/articl e/view/11043.
16. Silveira DS, Santos IS, Costa JSD. Atenção pré-natal na rede básica: uma avaliação da estrutura e do processo. Saúde Pública (Rio de Janeiro) [periódico na Internet]. 2016 [citado 2017 jun. 09]; 17(1): 1-9. Disponível em: http://www.scielo.br/pdf/csp/v 17n1/4068.pdf.

17. Dias-da-Costa JS, Cesar JA, Haag CB, Watte G, Vicenzi K, Schaefer R. Inadequação do pré-natal em áreas pobres no Nordeste do Brasil: prevalência e alguns fatores associados. Saúde Materno Infantil (Recife) [periódico na Internet]. 2013 [citado 2017 jun. 29]; 2(13): 101-09. Disponível em: http://www.scielo.br/ pdf/rbsmi/v13n2/a03v13n2.pdf.

18. Leal MC, Theme-Filha MM, Moura EC, Cecatti JG, Santos LMP. Atenção ao pré-natal e parto em mulheres usuárias do sistema público de saúde residentes na Amazônia Legal e no Nordeste, Brasil 2010. Saúde Materno Infantil (Recife) [periódico na Internet]. 2015 [citado 2017 jul. 09]; 1(15): 91-04. Disponível em: http://www.scielo.br/scielo.php?pid=S 1519-38292015000100091\&script $=$ sci _abstract\&tlng $=$ pt.

19. Souza AMM, Souza KV, Rezende EM, Martins EF, Campos D, Lansky S. Práticas na assistência ao parto em maternidades com inserção de enfermeiras obstétricas, em Belo Horizonte, Minas Gerais. Enfermagem (Rio de Janeiro) [periódico na Internet]. 2015 [citado 2017 jul. 16]; 20(2):324-31. Disponível em: http://www.scielo.br/pdf/ean/v20n2/14 14-8145-ean-20-02-0324.pdf. 
20. Davis-floyd R. The technocratic, humanistic and holistic paradigms of childbirth. Int J Gynaecol Obstet. 2001; 75(1):S5-S23.

21. Ministério da Saúde (BR). Secretaria de Atenção à Saúde. Atenção ao pré-natal de baixo risco. Departamento de atenção básica. Brasília: Editora do Ministério da Saúde; 2012.

22. Oliveira JDG, Campo TNC, Souza FMLC, Davim RMB, Dantas JC. Percepção de enfermeiros obstetras na assistência à parturiente. Enfermagem (Recife) [periódico na Internet]. 2016 [citado 2017 ago. 11]; 10(10):3868-75. Disponível em: https://periodicos.ufpe. br/revistas/revistaenfermagem/article/d ownload/11454/13277.

23. Word Health Organization. Care in normal birth: a practical guide: report of a Technical Working Group. Geneve: WHO; 1996.

24. Carvalho VF, Kerber NPC, Azambuja EP, Bueno FF, Silveira RS, Barros AM. Direitos das parturientes: conhecimento da adolescente e acompanhante. Saúde e Sociedade (São Paulo) [periódico na Internet]. 2014 [citado 2017 ago. 18]; 23(2), 572-81. Disponível em: http://www. scielo.br/pdf/sausoc/v23n2/0104-1290sausoc-23-2-0572.pdf.

25. Carneiro RG. Dilemas antropológicos de uma agenda de saúde pública: programa Rede Cegonha, pessoalidade e pluralidade. Saúde e Educação (Botucatu) [periódico na Internet]. 2013 [citado 2017 ago. 22];17(44)49-9. Disponível em: http://www.scielo.br/pdf/icse/v17n 44/a05v17n44.pdf.
26. Ministério da Saúde (BR). Instituição da Política Nacional de Atenção Obstétrica e Neonatal. Portaria $n^{\circ} 1067$ de 4 de julho de 2005. Diário Oficial da República Federativa do Brasil. Brasília: Ministério da Saúde; 2005.

27. Cavalcanti PCS, Junior GDG, Vasconcellos ALR, Guerrero AVP. Saúde Coletiva (Rio de Janeiro) [periódico na Internet]. 2013 [citado 2017 ago. 17]; 23(4):1297-316. Disponível em: http://www.scielo.br/s cielo.php? script $=$ sci_arttext\&pid $=\mathrm{S} 010$ 3-73312013000400014. 